\title{
Comparison between the lattice dynamics and molecular dynamics methods: calculation results for $\mathrm{MgSiO}_{3}$ perovskite
}

\author{
Masanori Matsui \\ Department of Earth and Planetary Sciences, Faculty of Science, Kyushu University \\ Geoffrey D. Price and Atul Patel \\ Research School of Geological and Geophysical Sciences, Birkbeck College and University College \\ London
}

\begin{abstract}
The lattice dynamics (LD) and molecular dynamics (MD) methods have been used to calculate the structure, bulk modulus, and volume thermal expansivity of $\mathrm{MgSiO}_{3}$ perovskite, in order to investigate the reliability of the two simulation techniques over a wide range of temperature and pressure conditions. At an intermediate temperature of $500 \mathrm{~K}$ and zero pressure, the $\mathrm{LD}$ and $\mathrm{MD}$ values are in exellent agreement for both the structure and bulk modulus of $\mathrm{MgSiO}_{3}$ perovskite. At high temperatures and zero pressure, however, the LD method, which is based on the quasi-harmonic approximation, increasingly overestimates the molar volume of $\mathrm{MgSiO}_{3}$ perovskite because of the neglect of higher-order anharmonic terms. At the high temperatures and high pressures prevailing in the lower mantle, the errors in the $\mathrm{LD}$ values for both the molar volume and bulk modulus, relative to the MD values, are generally small or negligible. However, since anharmonicity decreases substantially with pressure but increases rapidly with temperature, the error in the LD simulated volume thermal expansivity is serious, especially in the lower pressure region.
\end{abstract}

\section{Introduction}

The detailed description of the chemical and physical properties of the Earth's interior requires an accurate knowledge of the elastic and thermal properties of the constitutive minerals at high temperatures and high pressures. However, it is still extremely difficult to carry out experiments to measure accurately such properties at the combined temperature and pressure conditions to be found in the Earth's deep interior. As an alternative to direct experimental study, therefore, atomistic computer simulations have recently been extensively applied to predict the properties of various minerals thought to exist in the interior of the Earth [e.g. Wolf and Bukowinski, 1985; Cohen, 1987; Price et al., 1987; Matsui, 1988; Matsui and Price, 1992].

Given a set of interatomic potentials, there are two major types of simulation techniques that can be used to calculate static and dynamic properties of crystals at a specified temperature $T$ and pressure $P$, namely the lattice dynamics (LD) and molecular dynamics (MD) methods.

Copyright 1994 by the American Geophysical Union.

Paper number 94GL01370

0094-8534/94/94GL-01370\$03.00
In the $\mathrm{LD}$ method the crystal potential is usually estimated in the harmonic or quasi-harmonic approximation. LD calculations are very useful and powerful for predicting vibrational frequencies and thermodynamic properties of crystals over a wide temperature range, but break down at very high temperatures [Price et al., 1987; Parker and Price, 1989]. For such high temperatures, where anharmonicity is important and quantum effects are small, it is generally more practicable to use the MD method. In this method the average structural parameters are simulated by using Newton's classical equations of motion for atoms in crystals, as a function of time. The MD method can be applied to predict not only equilibrium properties [e.g. Matsui, 1988; Matsui and Price, 1992] but also time-dependent phenomena, such as first-order phase transitions [Tsuneyuki et al., 1989] and transport properties [Wall and Price, 1989].

$\mathrm{MgSiO}_{3}$ perovskite is thought to be the major component of the lower mantle. The $\mathrm{LD}$ and $\mathrm{MD}$ methods have therefore been extensively applied to simulating the structural and physical properties of this phase. Examples of the application of the LD method to the simulations of $\mathrm{MgSiO}_{3}$ perovskite include the calculation of the equation of state [Hemley et al., 1987], and the establishment of constraint on mineral composition models of the lower mantle from simulated structural and elastic properties of $\mathrm{MgSiO}_{3}$ perovskite [Cohen, 1987; Bukowinski and Wolf, 1988]. The MD technique has been used to predict the possible existence of a high temperature and/or high pressure phase transition in $\mathrm{MgSiO}_{3}$ perovskite [Matsui and Price, 1991; Kapusta and Guillopé, 1993].

The aim of the present investigation is to compare the values computed using the $\mathrm{LD}$ and $\mathrm{MD}$ techniques for the structure, molar volume, $V$, volume thermal expansivity, $\alpha$, and isothermal bulk modulus, $K_{\mathrm{T}}$, of $\mathrm{MgSiO}_{3}$ perovskite, and to assess the reliability and applicability of the two methods over a wide temperature and pressure range, with special reference to the simulation of lower mantle conditions.

\section{Calculation methods}

All the $\mathrm{LD}$ and $\mathrm{MD}$ calculations were made using the pair potential MAM0K [Matsui, 1988], which has been shown to reproduce the observed structural and physical properties of $\mathrm{MgSiO}_{3}$ perovskite quite accurately [Matsui and Price, 1992]. The LD calculations were carried out using the PARAPOCS computer code, which obtains the 
equilibrium structure by minimizing the free energy of the crystal with respect to the structural parameters, within the framework of the quasiharmonic approximation, as described in Parker and Price [1989]. The MD simulations were performed in the isothermal-isobaric ensemble, i.e., with fixed $T, P$, and number of ions. The equilibrium structural parameters were derived by taking averages over a sufficiently long time-interval, and quantum corrections to the classical $\mathrm{MD}$ values were made using the Wigner-Kirkwood expansion of the free energy in terms of Planck constant, by the technique proposed by Matsui [1989]. In the present MD work, we took a basic cell composed of $27(3 \mathbf{a} \times 3 \mathbf{b} \times 3 \mathbf{c})$ unit cells, containing 540 atoms. In an attempt to estimate the sensitivity of the system to ensenble size, we also performed MD calculations with a larger basic cell containing 960 atoms. Simulations with such an ensenble carried out at 500, 1000 and $1500 \mathrm{~K}$ under zero pressure were compared with the calculated values of $V$ and $\alpha$ obtained for the system composed of 540 atoms. Within the limit of statistical uncertainties, we found no significant system size effect.

$\mathrm{MgSiO}_{3}$ perovskite is orthorhombic with space group Pbnm [Horiuchi et al., 1987]. We note, in each of our LD and MD calculations, no symmetry constraint was imposed either on the cell parameters or on the atomic coordinates in the unit cell. The $K_{\mathrm{T}}$ 's, were obtained by fitting the simulated $P-V$ relations to a third-order BirchMurnaghan equation, while the $\alpha$ 's were derived by fitting the computed $T-V$ results to the equation $\alpha=(1 / V)(\partial V / \partial T)$ $=\alpha_{0}+\alpha_{1} T+\alpha_{2} T^{-2}$, where $\alpha_{0}, \alpha_{1}$ and $\alpha_{2}$ are the coefficients of thermal expansion.

\section{Results and discussion}

Since the MD method is exact in the high temperature limit, then when combined with quantum corrections through the Wigner-Kirkwood expansion, it is the best approach to determining properties at high temperatures. On the other hand the LD technique, with the quasiharminic approximation, is accurate at lower temperatures, where anharmonic effects are negligible.
Table 1. $\mathrm{LD}$ and $\mathrm{MD}$ simulated structures of $\mathrm{MgSiO}_{3}$ perovskite at $500 \mathrm{~K}$ and zero pressure.

\begin{tabular}{|c|c|c|c|c|c|}
\hline & LD & MD & & LD & MD \\
\hline \multicolumn{2}{|c|}{ Cell parameters } & \multicolumn{4}{|c|}{$M g-O$ distances/A } \\
\hline$a / \mathrm{A}$ & 4.77 & 4.78 & $\mathrm{Mg}-\mathrm{O} 1$ & 2.00 & 2.00 \\
\hline$b$ & 4.93 & 4.93 & $-\mathrm{O} 2[2]$ & 2.05 & 2.05 \\
\hline \multirow[t]{2}{*}{$c$} & 6.97 & 6.95 & $-\mathrm{O} 1$ & 2.13 & 2.13 \\
\hline & & & $-02[2]$ & 2.29 & 2.30 \\
\hline \multicolumn{2}{|c|}{ Si-O distances/A } & & $-\mathrm{O} 2[2]$ & 2.48 & 2.47 \\
\hline $\mathrm{Si}-\mathrm{O} 2[2]^{\dagger}$ & 1.78 & 1.79 & -01 & 2.85 & 2.85 \\
\hline-02 [2] & 1.79 & 1.79 & -01 & 2.93 & 2.92 \\
\hline$-01[2]$ & 1.82 & 1.81 & $-\mathrm{O} 2[2]$ & 3.09 & 3.08 \\
\hline$<\mathrm{Si}-\mathrm{O}\rangle$ & 1.80 & 1.80 & $<\mathrm{Mg}-\mathrm{O}>$ & 2.48 & 2.48 \\
\hline
\end{tabular}

${ }^{\dagger}$ Multiplicity of bond

The Debye temperature of $\mathrm{MgSiO}_{3}$ perovskite is reported to be about $1000 \mathrm{~K}$ at $0 \mathrm{GPa}$ [Akaogi and Ito, 1993]. We first made a comparison between the $\mathrm{LD}$ and $\mathrm{MD}$ results, therefore, at intermediate conditions, $500 \mathrm{~K}$ and 0 $\mathrm{GPa}$, under which both methods are expected to be valid. Table 1 gives the $\mathrm{LD}$ and $\mathrm{MD}$ calculated cell parameters and interatomic distances for $\mathrm{MgSiO}_{3}$ perovskite at these conditions. We find that at $500 \mathrm{~K}$ and $0 \mathrm{GPa}$ the simulated structural data for the two methods are in excellent agreement. The maximum error in the cell parameters is only $0.2 \%$, and the errors in the interatomic distances are within only $0.01 \mathrm{~A}$. The isothermal bulk modulus at $500 \mathrm{~K}$ and $0 \mathrm{GPa}$, calculated by $\mathrm{LD}$, is 237 $\mathrm{GPa}$, which is again in excellent agreement with the value obtained from MD of $236 \mathrm{GPa}$ (see Table 2). These agreements, in both the structure and bulk modulus, are quite satisfactory, considering the greatly different nature of the two calculation methods.

Fig. 1 shows the temperature dependence of the simulated molar volumes of $\mathrm{MgSiO}_{3}$ perovskite at zero pressure. As expected, the LD-simulated molar volume increases more rapidly with temperature than the $\mathrm{MD}$

Table 2. $\mathrm{LD}$ and $\mathrm{MD}$ calculated values for the molar volume, $V$, volume thermal expansivity, $\alpha$, and isothermal bulk modulus, $K_{\mathrm{T}}$, of $\mathrm{MgSiO}_{3}$ perovskite at selected temperatures $T$ and pressures $P$.

\begin{tabular}{|c|c|c|c|c|c|c|c|c|}
\hline \multirow[t]{2}{*}{$T / \mathrm{K}$} & \multirow[t]{2}{*}{$P / G P a$} & \multicolumn{2}{|c|}{$V /\left(\mathrm{cm}^{3} / \mathrm{mol}\right)$} & \multirow{2}{*}{$\begin{array}{c}\text { error } \\
\%\end{array}$} & \multicolumn{2}{|c|}{$\alpha /\left(10^{-5} \mathrm{~K}^{-1}\right)$} & \multicolumn{2}{|c|}{$K_{\mathrm{T}} / \mathrm{GPa}$} \\
\hline & & $\mathrm{LD}$ & MD & & LD & MD & LD & MD \\
\hline 500 & 0 & 24.68 & 24.70 & 0.1 & $3.75(7)^{\dagger}$ & $3.31(19)$ & $237(1)$ & $236(1)$ \\
\hline 1000 & 0 & 25.25 & 25.19 & 0.2 & $5.32(9)$ & $4.38(20)$ & $200(2)$ & $208(1)$ \\
\hline 500 & 30 & 22.35 & 22.37 & 0.1 & $2.02(4)$ & $1.88(18)$ & $378(1)$ & $377(2)$ \\
\hline 1000 & 30 & 22.60 & 22.60 & 0.0 & $2.36(4)$ & $2.24(10)$ & $354(3)$ & $356(1)$ \\
\hline 2000 & 30 & 23.21 & 23.16 & 0.2 & $3.05(5)$ & $2.59(11)$ & $304(6)$ & $314(4)$ \\
\hline 3000 & 30 & 24.02 & 23.80 & 0.9 & $3.73(7)$ & $2.89(13)$ & $251(17)$ & $279(5)$ \\
\hline 500 & 60 & 20.88 & 20.89 & 0.0 & $1.37(3)$ & $1.26(12)$ & $509(2)$ & $509(3)$ \\
\hline 1000 & 60 & 21.04 & 21.05 & 0.0 & $1.68(2)$ & $1.61(7)$ & $496(6)$ & $493(2)$ \\
\hline 2000 & 60 & 21.43 & 21.41 & 0.1 & $1.94(2)$ & $1.79(8)$ & $453(11)$ & $455(7)$ \\
\hline 3000 & 60 & 21.87 & 21.81 & 0.3 & $2.16(2)$ & $1.92(9)$ & $402(30)$ & $417(8)$ \\
\hline
\end{tabular}

+ Parenthesized figures refer to the estimated standard deviation of least units cited. 


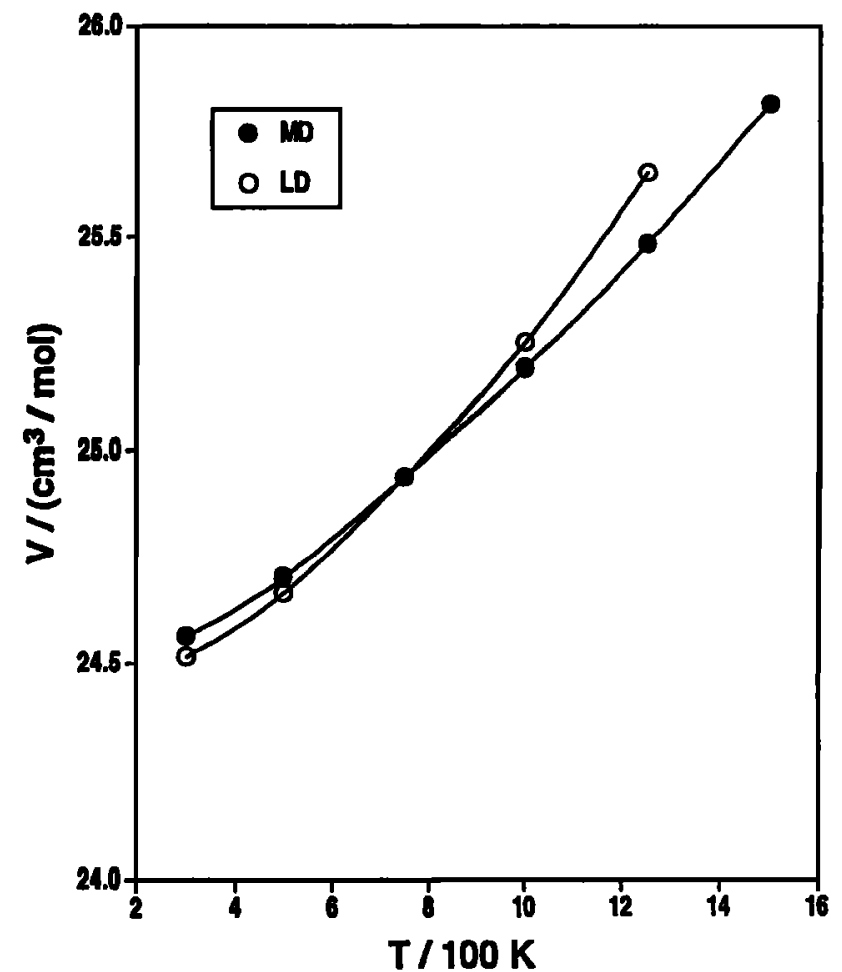

Figure 1. Temperature dependencies of the $\mathrm{LD}$ and $\mathrm{MD}$ computed molar volumes of $\mathrm{MgSiO}_{3}$ perovskite at zero pressure.

volume, due to the neglect of higher-order anharmonic terms in the LD calculations at high temperature. The overestimation of the $\mathrm{MD}$ molar volumes relative to the LD results, at temperatures lower than approximately 700 $\mathrm{K}$, might be explained by the neglect of higher-order quantum corrections in our $\mathrm{MD}$ simulations, as described previously [Matsui, 1989]. It is to be noted that although the difference in the $\mathrm{LD}$ and $\mathrm{MD}$ simulated molar volumes is generally very small, e.g. only 0.1 and $0.2 \%$ at 500 and $1000 \mathrm{~K}$ respectively, the difference in the calculated volume thermal expansivities is significant, and reaches 22 $\%$ at $1000 \mathrm{~K}$ (see Table 2).

Above approximately $1500 \mathrm{~K}$ at zero pressure, the quasi-harmonic LD calculations indicate the orthorhombic $\mathrm{MgSiO}_{3}$ perovskite structure to be dynamically unstable, with imaginary mode-frequencies, in accord with similar quasi-harmonic LD simulations of $\mathrm{MgSiO}_{3}$ perovskite by Wolf and Bukowinski [1985], who used a modified electron gas potential. However, it is important to note that this dynamical instability is solely the result of the break down in quasi-harmonic approximation. Actually, in our $\mathrm{MD}$ simulations, the orthorhombic $\mathrm{MgSiO}_{3}$ perovskite structure is found to remain stable at all temperatures up to the melting point (calculated to be about $2800 \mathrm{~K}$ at zero pressure), as described previously [Matsui and Price, 1991].

Accurate data on $V, \alpha$ and $K_{\mathrm{T}}$ for $\mathrm{MgSiO}_{3}$ perovskite at high temperatures and high pressures are necessary in order to estimate detailed chemical and physical properties of the lower mantle. Table 2 lists the $\mathrm{LD}$ and MD computed values for $V, \alpha$, and $K_{\mathrm{T}}$ at $T$ from 500 to 3000 $\mathrm{K}$, and $P$ of 30 and $60 \mathrm{GPa}$, which correspond approximately to depths of 800 and $1450 \mathrm{~km}$ in the lower mantle [Dziewonski and Anderson, 1981]. Fig. 2 illustrates the calculated molar volumes of $\mathrm{MgSiO}_{3}$ perovskite from the two simulations as a function of pressure up to $100 \mathrm{GPa}$, for the two temperatures 2000 and $3000 \mathrm{~K}$, which can be thought to be typical for the lower mantle [Spiliopoulos and Stacey, 1984; Jeanloz and Morris, 1986]. The differences between the LD and MD values at high temperatures show the magnitude of the error in the LD calculations which results from the neglect of explicit anharmonic terms. In Fig. 2 and Table 2 we see the difference between the $\mathrm{LD}$ and $\mathrm{MD}$ results. At 30 $\mathrm{GPa}$ for example, the $\mathrm{LD}$ and $\mathrm{MD}$ values for $\alpha$ agree within uncertainties at $1000 \mathrm{~K}$, however the values diverge by $18 \%$ at $2000 \mathrm{~K}$, and by $27 \%$ at $3000 \mathrm{~K}$. From Fig. 2 we also see that anharmonicity decreases substantially with increasing pressure; for both 2000 and $3000 \mathrm{~K}$ simulations, the LD values overestimate $V$ at lower pressures, however the extent of these overestimations decrease with pressure, such that the LD values eventually agree with the MD results within statistical uncertainties at the highest pressures.

At typical $T$ and $P$ conditions in the lower mantle, the deviations of the LD results from those of MD for both $V$ and $K_{\mathrm{T}}$ are generally small or negligible. However, since anharmonicity changes substantially with pressure and even more with temperature, the deviation in $\alpha$ is quite significant, especially in the lower pressure region in the lower mantle. In conclusion, therefore we believe that if accurate calculations of the physical properties (e.g. thermal expansion coefficient) of minerals under lower mantle conditions are required, full molecular dynamic simulations must be performed, or corrections to the quasi-harmonic approximation must be applied to lattice

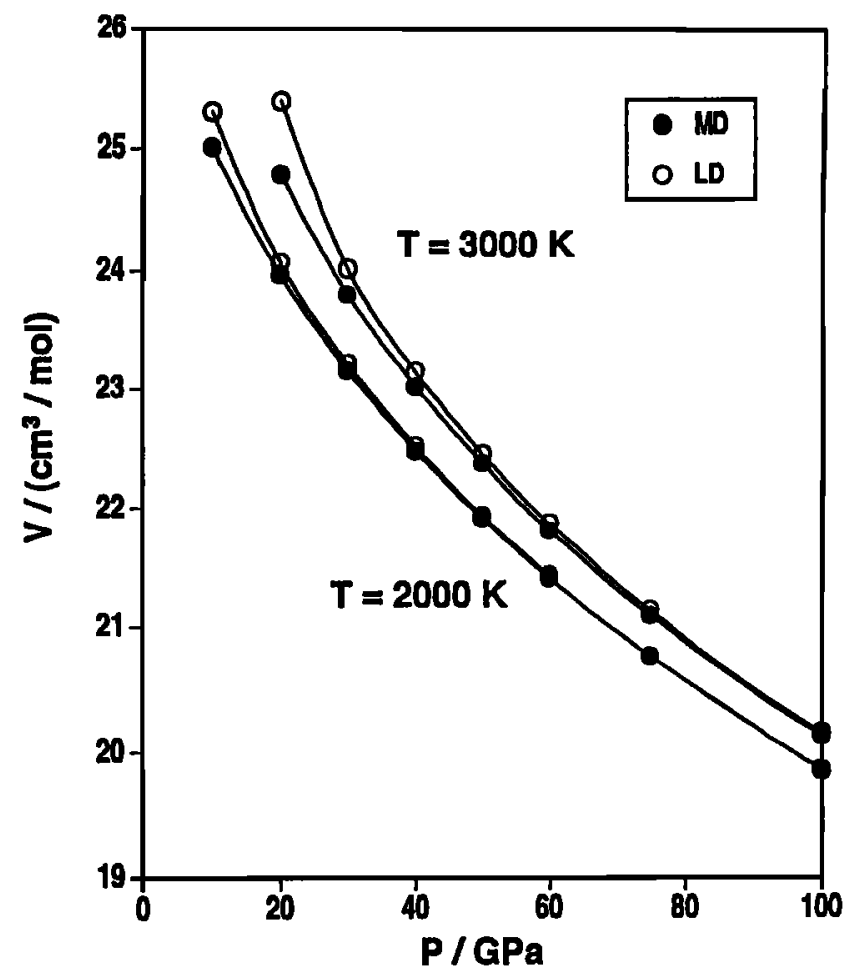

Figure 2. The LD and MD simulated molar volumes of $\mathrm{MgSiO}_{3}$ perovskite at the temperatures 2000 and $3000 \mathrm{~K}$ as a function of pressure up to $100 \mathrm{GPa}$. 
dynamical simulations to take full account of intrinsic anharmonic effects.

Acknowledgements. We are grateful to M. S. T. Bukowinski and an anonymous reviewer for constructive and useful comments. This research is supported by the NERC (GR3/6970) to G.D.P., and by Grants-in-Aid for Scientific Research from Ministry of Education, Science and Culture to M.M.

\section{References}

Akaogi, M. and E. Ito, Heat capacity of $\mathrm{MgSiO}_{3}$ perovskite, Geophys. Res. Lett., 20, 105-108, 1993.

Bukowinski, M. S. T. and G. H. Wolf, Equation of state and posible critical phase transitions in $\mathrm{MgSiO}_{3}$ perovskite at lower-mantle conditions, in Structural and Magnetic Phase Transitions in Minerals edited by S. Ghose, J. M. D. Coey, and E. Salje, Springer-Verlag, New York, 1988.

Cohen, R. E., Elasticity and equation of state of $\mathrm{MgSiO}_{3}$ perovskite, Geophys. Res. Lett., 14, 1053-1056, 1987.

Dziewonski, A. M. and D. L. Anderson, Preliminary reference Earth model, Phys. Earth Planet. Inter. 25, 297-356, 1981.

Hemley, R. J., M. D. Jackson, and R. G. Gordon, Phys. Chem. Minerals, 14, 2-12, 1987.

Horiuchi, H., E. Ito, and D. J. Weidner, Perovskite-type $\mathrm{MgSiO}_{3}$ : single-crystal X-ray diffraction study, $\mathrm{Am}$. Mineral., 72, 357-360, 1987.

Jeanloz, R. and S. Morris, Temperature distribution in the crust and mantle, Ann. Rev. Earth Planet. Sci., 14, 377415,1986

Kapusta, B. and M. Guillopé, Molecular dynamics study of the perovskite $\mathrm{MgSiO}_{3}$ at high temperature: structural, elastic and thermodynamical properties, Phys. Earth Planet. Inter., 75, 205-224, 1993.

Matsui, M., Molecular dynamics study of $\mathrm{MgSiO}_{3}$ perovskite, Phys. Chem. Minerals, 16, 234-238, 1988.
Matsui, M., Molecular dynamics study of the structural and thermodynamic properties of $\mathrm{MgO}$ crystal with quantum correction, J. Chem. Phys., 91, 489-494, 1989.

Matsui, M. and G. D. Price, Simulation of the premelting behaviour of $\mathrm{MgSiO}_{3}$ perovskite at high pressures and temperatures, Nature, 351, 735-737, 1991.

Matsui, M. and G. D. Price, Computer simulation of the $\mathrm{MgSiO}_{3}$ polymorphs, Phys. Chem. Minerals, 18, 365-372, 1992.

Parker, S. C. and G. D. Price, Computer modelling of phase transitions in minerals, Advances in Solid-State Chemistry, 1, 295-327, 1989.

Price, G. D., S. C. Parker, and M. Leslie, The lattice dynamics and thermodynamics of the $\mathrm{Mg}_{2} \mathrm{SiO}_{4}$ polymorphs, Phys. Chem. Minerals, 15, 181-190, 1987.

Spiliopoulos, S. and F. D. Stacey, The Earth's thermal profile: is there a mid-mantle thermal boundary layer? $J$. Geodyn., 1, 61-77, 1984.

Tsuneyuki, S., Y. Matsui, H. Aoki, and M. Tsukada, New pressure-induced structural transformations in silica obtained by computer simulation, Nature, 339, 209-211, 1989.

Wall, A. and G. D. Price, Electrical conductivity of the lower mantle: a molecular dynamics simulation of $\mathrm{MgSiO}_{3}$ perovskite, Phys. Earth Planet. Inter., 58, 192-204, 1989.

Wolf, G. H. and M. S. T. Bukowinski, Ab initio structural and thermoelastic properties of orthorhombic $\mathrm{MgSiO}_{3}$ perovskite, Geophys. Res. Lett., 12, 809-812, 1985.

M. Matsui, Department of Earth and Planetary Sciences, Faculty of Science, Kyushu University, Hakozaki, Fukuoka 812, Japan.

G. D. Price and A. Patel, Research School of Geological and Geophysical Sciences, Birkbeck College and University College London, Gower Street, London, WC1E 6BT, England.

(Received March 17, 1994; revised April 18, 1994; accepted May 17, 1994) 